\title{
Newborn After Prolonged Leakage of Liquor Amnii
}

\author{
A. D. BAIN,* M.D.; I. I. SMITH,* M.B., CH.B.; I. K. GAULD,* A.I.M.L.T.
}

Brit. med. F., 1964, 2, 598-599

The continued elucidation of the trisomic syndromes with their varied and complex external abnormalities has emphasized the necessity for skilled recognition of significant deviations from normal in the outward appearance of the newborn. Mongolism, trisomy 17-18, trisomy 13-15, the recognized autosomal trisomic syndromes, show an odd facial appearance and abnormalities of the hands, feet, and ears.

For some time we have been aware of a number of perinatal deaths with which certain external abnormalities were so often involved that they appeared to form a distinct group. Over the past few years 20 babies of this group have been systematically photographed, subjected to detailed post-mortem examination, and chromosome studies undertaken on the more recent cases.

\section{Investigations}

The 20 perinatal deaths comprised 8 stillbirths and 12 neonatal deaths. The longest period of survival was 18 hours. In all cases there was an abnormal facial appearance, the predominating feature being flattening of the tip of the nose. What we thought to be a significant abnormality in the ears was present in 10 of these babies ; this consisted of flattening of the ears against the head. The presence of abnormal epicanthic folds was evident in the majority. Short great toes were present in three of these cases. In addition four showed varus deformity of the feet. The hands were unusually broad in three cases, but in the remainder they were normal.

The average weight of these babies was $1,350 \mathrm{~g}$., with a range of $1,100-1,850 \mathrm{~g}$. The gestational age varied from 27 to 35 weeks, but a correlation of the menstrual delivery period and birth weight showed no deviation from normal.

Post-mortem Examination.-The cause of death in the 12 live-born infants was pulmonary hyaline membrane in four, pulmonary haemorrhage in one, and intraventricular cerebral haemorrhage in two; in the remaining five there was no satisfactory explanation other than prematurity. Pneumonia was present in three instances, all associated with hyaline membrane. No internal congenital malformations were noted either in stillbirths or in neonatal deaths. The kidneys and urinary tracts were normal.

Chromosome Analysis.-Successful lymphocyte cultures were prepared from thymus and spleen in four instances, and in two of these fibroblast cultures were also prepared from pericardium and lung. Analysis of the chromosomes revealed, in each case, a modal number of 46 with a normal karyotype.

Mode of Presentation.-Presentation was breech in eight cases, transverse in one case, and vertex in 11 cases.

Maternal Histories. - The following clinical observations on the volume of liquor were made in the maternal case notes: definite deficiency in the volume of liquor was specifically mentioned in eight cases, probable deficiency could be inferred in four, and there was no record in the remaining eight. Further scrutiny of the case records, however, revealed a definite occurrence of ante-partum leakage of liquor in 16 of the 20 cases. In a further three there was a definite history of continued loss of blood without specific mention of liquor,

\footnotetext{
* Departments of Pathology, the Royal Hospital for Sick Children and
} the University of Edinburgh. and in the remaining case record, while there was no suggestion of loss of liquor or blood, oligohydramnios was suspected. In cases with a specific history of loss of liquor the period over which the loss was noted varied from 3 to 19 weeks.

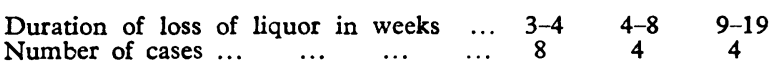

Antenatal radiographic examination was carried out in one instance on account of prolonged leakage of liquor and the low height of the fundus in relation to the gestational age. $X$-ray films showed the foetus to be hyperflexed, and the radiologist suggested that this might be due to intrauterine death. The foetus, however, was certainly alive up to delivery one week later. Jeffcoate and Scott (1959) have already drawn attention to the possibility of this misinterpretation in cases of oligohydramnios.

Placental Examination.-The placenta was available for pathological examination in 11 of the 20 cases, and in nine of these it was circumvallate. The amniotic membranes in all 11 placentae were carefully examined for amnion nodosum. Absence of this condition on naked-eye examination was confirmed microscopically.

\section{Discussion}

The characteristic facial appearance first reported by Potter (1946a, 1946b) in babies with bilateral renal agenesis (Fig. 1)

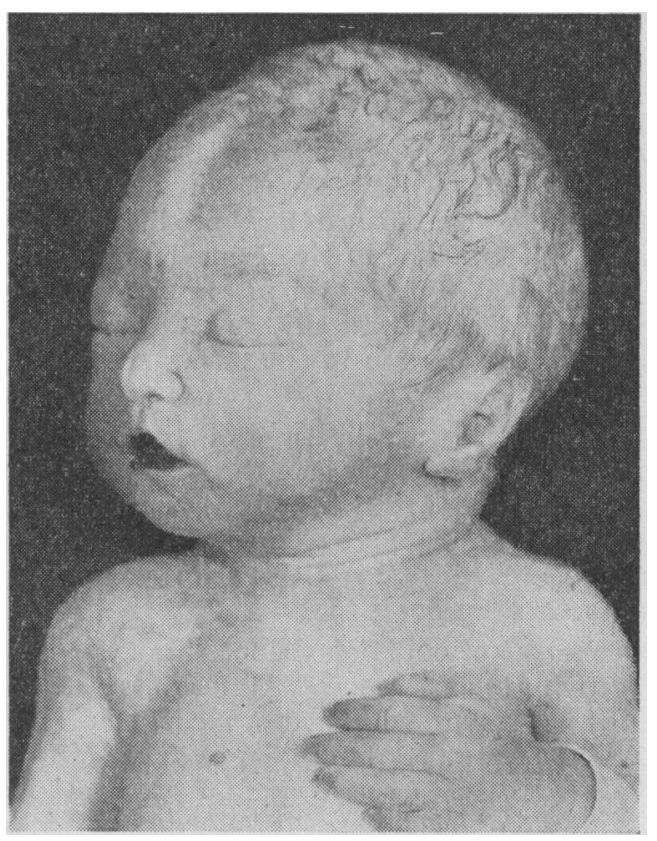

FIG. 1.-Facial appearance simulating that described by Potter.

focused attention on the importance of recognizing external abnormalities in the newborn. That the so-called Potter facies could also be found in association with any renal or urinarytract anomaly resulting in failure of foetal micturition in utero was noted by Bain and Scott (1960) and subsequently confirmed by Blanc (1961) and Kohler (1961). Bain and Scott 
also drew attention to the similar significance of abnormal broadening of the hands.

The high incidence of anhydramnios or oligohydramnios in such cases has suggested a simple mechanical explanation for the presence of the abnormal facies and the other associated anomalies involving hands, feet, and ears. Experience has led us to believe that although Potter's original description is invariably specific in relation to bilateral renal agenesis, in the instances of oligohydramnios due to causes other than foetal there is in fact a wide variation in the extent to which any single feature in the face, hands, feet, or ears may be affected. That these features can be misinterpreted in the premature baby is demonstrated by the fact that a clinical diagnosis of renal agenesis had already been made in two out of the present series of cases, yet in neither was an abnormality of the kidney or urinary tract found at necropsy. A history of prolonged leakage of liquor would have been of considerable clinical help in excluding a renal anomaly in the infants. It is significant that retrospective examination of the case records showed such a history.

Placental examination in our series revealed a high incidence of severe circumvallation (Fig. 2). Scott (1960), in a review

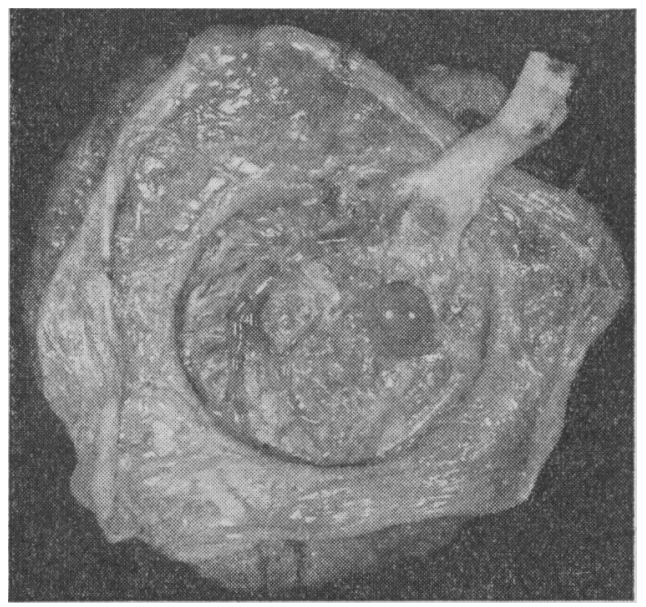

FIG. 2.-Placenta from baby in Fig. 1, showing a severe degree of circumvallation.

of circumvallate placentae, cites previous authors who commented on the association of this placental abnormality with " hydrorrhoea gravidarum," presumably leaking liquor. Amnion nodosum, a feature suggestive of oligohydramnios, was absent in our cases but has in fact been observed in two other instances of circumvallate placenta associated with prolonged leakage of liquor (Scott, personal communication, 1963). In a review of amnion nodosum Blanc et al. (1962) describe this condition in association with prolonged leakage of liquor, several of the babies showing not only the Potter facies but abnormal ears and the equinovarus deformity. None of these babies had any renal anomaly.

Short great toes, one of the features generally associated with trisomy $17-18$, were present in 3 out of 20 babies in the present review. In our experience short great toes and some degree of apparent flexion deformity of the fingers are not an uncommon finding in otherwise normal very premature babies.
It seems probable, therefore, that, as in bilateral renal agenesis where compression of the foetus secondary to oligohydramnios accounts for the facial features, in prolonged leakage of liquor compression of the foetus may also occur, but the features produced in the baby are more variable and are dependent on the duration and extent of the leakage of liquor. Support for this view is to be found in the work of Bearn (1961), who stated that in mammalian foetuses subjected to surgical interference and allowed to continue to term, severe compression and a snakelike appearance of the foetus were observed. He also noted that this deformity occurred particularly after too much amniotic fluid had escaped during the operation and when the incision in the internal wall had failed to heal and allowed amniotic fluid to leak.

\section{Prognosis}

It would appear that the prognosis in babies born after prolonged leakage of liquor is not favourable. Of those not stillborn the majority present with respiratory difficulty. In some instances this might in fact be related to pulmonary hypoplasia. It is recognized that pulmonary hypoplasia is found in association with bilateral renal agenesis and other severe abnormalities of the kidney and urinary tract, and is therefore, in these abnormalities, related to the oligohydramnios. In the present series, where oligohydramnios was established, post-mortem examination suggested the presence of pulmonary hypoplasia, but confirmatory histological evidence could not be obtained. This was due to the difficulty in interpreting the histological appearance of the lungs in the premature babies covering such a range of gestational ages and birth weights.

\section{Summary}

A group of babies is described showing features akin to those recorded by Potter in association with bilateral renal agenesis. This group showed similar anomalies involving the hands, feet, and ears. No kidney or urinary-tract anomaly was found at necropsy. In this group there had been prolonged leakage of liquor or evidence of oligohydramnios. It has been shown that there is an association between oligohydramnios and the appearance of the baby. Chromosome studies on these babies have shown no abnormality.

We are grateful to the Distillers Co. Ltd. for a grant which has enabled us to further our work on congenital abnormalities; to the Board of Management, Royal Hospital for Sick Children, for providing facilities; and to Professors G. L. Montgomery and J. S. Scott for their interest and help.

\section{REFERENCES}

Bain, A. D., and Scott, J. S. (1960). Brit. med. F., 1, 841.

Bearn, J. G. (1961). Lancet, 1, 1058.

Blanc, W. A. (1961). N.Y. St. F. Med., 61, 1492.

- Apperson, J. W., and McNally, J. (1962). Bull. Sloane Hosp. Wom. (N.Y.), 8, 51 .

Jeffcoate, T. N. A., and Scott, J. S. (1959). Canad. med. Ass. F., 80, 77.

Kohler, H. G. (1961). Lancet, 1, 946.

Potter, E. L. (1946a). F. Pediat., 29, 68.

- (1946b). Amer. F. Obstet. Gynec., 51, 885.

Scott, J. S. (1960). F. Obstet. Gynaec. Brit. Cwlth, 67, 904. 